\title{
Psychological Health Assessment Model of Enterprise Employees Based on DNN Technology
}

\author{
Xin Li \\ School of Business, Macau University of Science and Technology, Macau 999078, China \\ Correspondence should be addressed to Xin Li; 1709853dbm30001@student.must.edu.mo
}

Received 14 November 2021; Revised 14 December 2021; Accepted 15 December 2021; Published 10 January 2022

Academic Editor: Xin Ning

Copyright (c) 2022 Xin Li. This is an open access article distributed under the Creative Commons Attribution License, which permits unrestricted use, distribution, and reproduction in any medium, provided the original work is properly cited.

\begin{abstract}
All enterprises gradually recognise the importance of employees' healthy psychology to business activities in order to improve their own economic level and occupy a certain leading position in the economic market. The main factors affecting employees' psychological health are used as input samples in this paper, and a network model of enterprise employees' psychological health prediction based on DNN is developed. To form a specific set, the psychological health indicators are separated from the complex test items. The key influencing factors in psychological health assessment are chosen as input vectors, and the DNN algorithm's output results are obtained, analysed, and compared. Following sample training, the artificial NN's error between predicted and measured values is only 3.55 percent, achieving the desired effect. The DNN principle is used in this paper to create a mathematical prediction network model based on an analysis of psychological factors affecting employees in businesses. The calculation of the final result of the prediction system is simple and flexible when the parameters of the NN are changed, and the network model's prediction efficiency and accuracy are greatly improved.
\end{abstract}

\section{Introduction}

The behaviour, psychology, and concept of employees are profoundly affected by intense work and fast-paced life in China, as a result of the continuous strengthening of social competition and the rapid development of the market economy [1]. In light of the current new development context, a link between employee psychological health issues and enterprise safety production has slowly emerged. We can only ensure the safe development of enterprise production activities by ensuring that employees have good psychological quality [2]. Employees with a healthy psychology have sufficient psychological endurance. They can significantly improve enterprise's competitiveness in the face of rising market competition [3]. Good psychological quality is an important guarantee for a successful career and a happy life as a group of employees who create enterprise wealth, but it is also an undeniable fact that an increasing number of employees are troubled by various psychological problems [4]. According to a survey, enterprise employees spend a significant amount of time in a high-pressure, unstable environment and are prone to negative psychological symptoms such as psychological tension and pain [5]. If the psychological health of employees has problems, it will directly have a serious impact on the safety production of the enterprise. Therefore, enterprises should pay attention to employees' psychological health problems, adopt diversified working methods, improve employees' psychological tolerance and anti frustration ability, ensure that enterprise safety production will not be affected by employees' psychological health problems, and promote the high-quality development of enterprises [6]. It has become a new topic for enterprise managers to timely alleviate employees' psychological pressure, promote employees' psychological health and build a harmonious enterprise [7].

Among the factors affecting the construction of a harmonious society, psychological health has become an important factor affecting the construction of a harmonious society. Individual psychological health is not only related to the healthy growth of individuals and the happiness of families but also related to the harmony of interpersonal relations, and even affect the construction of social morality, social responsibility, and social culture [8]. The evaluation of employees' psychological health behavior is an important 
content of enterprise management and enterprise EAP service. Judging from the current social situation, the psychological health problems of Chinese people are becoming more and more serious with the development of society. Extreme events caused by unhealthy mental state are frequent, and psychological health problems have had a certain negative impact on social harmony [9]. At present, the present situation of psychological health education for employees in enterprises is not optimistic, which mainly shows that a good cultural environment for psychological health education has not been established, the social support system for psychological health education is not perfect, and the government has not mobilized all sectors of society to participate in the work of psychological health education for employees [10]. At the individual level, employees do not pay enough attention to psychological health. Without the guidance of scientific knowledge, employees' psychological health problems cannot be scientifically analyzed and correctly handled, which is more likely to have adverse consequences [11]. In this paper, the main factors affecting the psychological health of enterprise employees are taken as the input samples, the characteristic psychological health indicators are separated from the complex test items, and the network model of enterprise employees' psychological health prediction system based on deep neural network (DNN) is established, so as to realize the prediction of enterprise employees' psychological health.

Mental health assessment and mental problem recognition are essentially pattern recognition or nonlinear classification problems. The psychological status of each individual is a multidimensional information system, which is characterized by multivariable, multilevel, and strong coupling. There are complex nonlinear interactions among various factors in the system. Therefore, it is difficult to describe it with traditional mathematical methods [12]. For perceptron model, its weight can be adjusted by learning, and the learning algorithm adopts supervised learning [13]. When it is used to classify two types of patterns, it is equivalent to separating two types of samples with a hyperplane in a highdimensional sample space. It has been proved that if the two input patterns are linearly separable sets, that is, there is a hyperplane that can separate them, then the algorithm must converge [14]. If the input pattern is a linear indivisible set, the single-layer perceptron cannot classify correctly. After the mathematical model is established, certain results can be deduced by logical reasoning or mathematical operation. If the model is given a certain explanation, the derived results can be regarded as some kind of prediction of the empirical system [15]. By further comparing the predicted value with the actual test value, the mathematical model can be modified according to the degree of agreement between them [16]. Based on the principle of DNN and the analysis of the psychological factors affecting employees, this paper establishes a mathematical prediction network model. By changing the parameters of the neural network (NN), the calculation of the final result of the prediction system is simple and flexible, and the prediction efficiency and accuracy of the network model are greatly improved. To a great extent, this provides a good reference method for the study of the psychological health status of enterprise employees.

\section{Related Work}

With the rapid development of social economy, the pace of people's life is constantly accelerating, and the pressure of life is also increasing. As employees' occupational health is the basic guarantee of enterprise safety production, enterprise managers should be aware that many problems caused by employees' physical and psychological health will have an impact on enterprise safety production. Literature [17] used SCL-90 as a measuring tool in the survey of enterprise employees, which showed that the psychological health status of female employees was significantly higher than that of male employees, regardless of each factor score or the total score of scale, that is, the psychological health status of female employees was poor. From the height of intelligent science, through combining with cognitive neuroscience, computer neuroscience, cognitive science, and so on, the research of NN has made breakthrough progress, and in the process of constantly exploring the essence of computational intelligence, the human brain $\mathrm{NN}$ is combined with DNN [18]. Literature [19] puts forward the fault prediction of different variables based on the established $\mathrm{NN}$ and carries out experiments on three technologies: linear analysis, recursive analysis, and genetic algorithm. The three technologies correspond to different variables, which are independent of each other. Literature [20] through the establishment of NN model and network learning, to a certain extent, provides the basis for the prediction of employees' psychological health. The evaluation model of psychological health established in literature [21] can judge psychological health well. Based on the fuzziness of psychological health and the highly nonlinear characteristics of psychological health evaluation, NN and fuzzy mathematics are effectively combined. Literature [9] takes the main influencing factors of employees' psychological health status as sample input, uses the optimized DNN to establish the prediction model of employees' psychological health status, takes the corresponding fuzzy comprehensive evaluation results as sample output, and uses the self-learning function of $\mathrm{NN}$ to train the network to get the mapping relationship between each factor and its psychological health status.

In this paper, the main factors affecting the psychological health of enterprise employees are taken as the input samples, the characteristic psychological health indicators are separated from the complex test items, and the network model of enterprise employees' psychological health prediction system based on DNN is established, so as to realize the prediction of enterprise employees' psychological health.

\section{Methodology}

3.1. Causes and Analysis of Psychological Problems of Enterprise Employees. Many factors can contribute to psychological issues. It will not only directly affect individual's mentality but will also be detrimental to the normal operation of enterprises [22] without scientific analysis and correct guidance in practical work. Employees in the workplace are frequently subjected to the pressures of elimination, professional title, and promotion, particularly in 
some assembly-line enterprises where the workload is heavy, the work standard is high, and there are numerous types of examinations, and they are in a constant state of high tension, which can put a strain on their physical and mental endurance. Based on an analysis of the problems and causes of enterprise employees' mental health psychological health education, practical countermeasures should be proposed to ensure the efficient operation of the cooperation mode by establishing a multilevel security system and creating a cooperation mode of enterprise employees' mental health psychological health education dominated by the government, enterprise subject, employees' participation, and social linkage. Many businesses use the task management mode of operation, focusing solely on the results of task completion rather than the psychological and emotional changes that occur during task completion. Employees with low work enthusiasm, high work pressure, absenteeism, high turnover, high accident rate, and tense interpersonal relationships are the result of many businesses failing to recognise the importance of maintaining employees' psychological health and failing to pay enough attention to their emotions. Burnout on the job refers to a state of physical, emotional, and psychological exhaustion [23]. If the persistent physical and mental exhaustion, pessimism, disappointment, boredom, and depression associated with burnout are not addressed in a timely manner, they will spread throughout the organisation, resulting in a depressed, negative, and pessimistic atmosphere. Employees are all working on the same project. When employees have conflicts with customers, partners, coworkers, or leaders, these conflicts and contradictions are not resolved in a timely manner, causing tension, psychological problems, and pressure, as well as affecting their work.

3.2. Basic Model of Psychological Health Assessment Based on $D N N$. Modern computers have strong information processing and computing capabilities, but their ability to make decisions, perception, pattern recognition [24-26], and other logical processing problems in complex environments is far less than that of people. They cannot think and learn and cannot change according to the changes of the environment, so they can only mechanically execute preprogrammed programs one by one. The computational response speed of each neuron in the human brain is very low, which cannot be compared with that of computers. However, due to the huge number of neurons, there are also many connections between neurons. As a result, the processing speed of many problems, especially logic problems, in the human brain is much faster than that of computers [27].

Because of the complexity and diversity of psychological phenomena, especially the interaction among various factors, the reliability of single-factor experiment results will be affected, so multifactor experiments and multistatistical methods are needed. Due to the complexity of the calculation process, the application of multivariate statistics was once restricted. In recent years, with the development and application of computers, the computational difficulties are gradually solved, and more and more multivariate statistical methods are cited by psychostatistics, thus promoting the

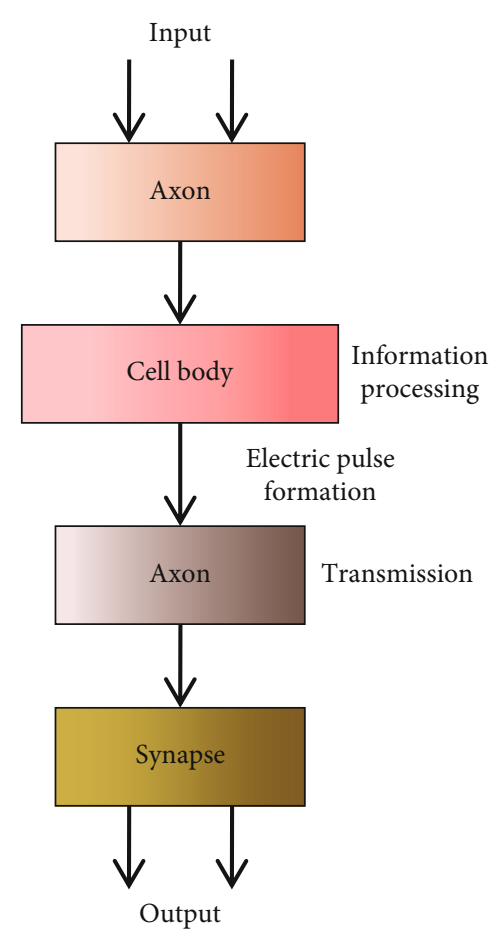

Figure 1: Basic model of NN.

development of psychostatistics. We cannot simply use a linear function to establish the prediction model of employees' psychological health status, because the process of each specific factor's function is invisible and intangible, and the influence on the results may be nonlinear, so we need a method that can reflect the corresponding relationship between the factors that affect the psychological status and the results to establish the prediction model. The basic model of NN is shown in Figure 1. The basic neuron model is shown in Figure 2.

DNN is a mathematical model for information processing which is connected by synapses similar to brain neurons [28]. The learning process of BP NN can be said to be an iterative process, which consists of forward propagation and backward propagation. The basic idea of the algorithm is to feed back the error of the output data from the output layer of the network and adjust the weights and thresholds of each layer of the NN step by step from the output layer, so as to minimize the mean square deviation of the output data of each sample [29].

Besides the characteristics of neural nodes, NNs also have the characteristics of topological structure.

Let $y_{k}$ be the actual network output of neuron $k$ at time $n$ when $x(n)$ is input, and $d_{k}(n)$ represents the output of the corresponding sample, then the error can be written as:

$$
e_{k}(n)=d_{k}(n)-y_{k}(n)
$$

Error correction learning is to make the objective function based on $e_{k}(n)$ reach the minimum, so that the actual output of each output neuron in the NN is statistically closest to the output of the sample. The most commonly used objective function is the criterion of mean square error, 


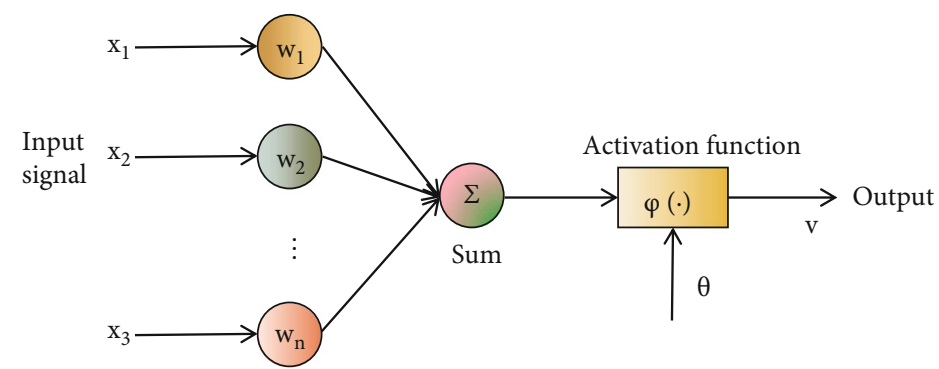

FIGURE 2: Basic neuron model.

which is defined as:

$$
J=E\left(\frac{\sum_{k} e_{k}^{2}(n)}{2}\right)
$$

where $E$ is the expectation operator. Because when $J$ is directly used as the objective function, it is necessary to count the characteristics of the whole process. In order to solve this difficulty, we generally replace $J$ with the instantaneous value $\varepsilon(n)$ of $J$ at time $n$, namely,

$$
\varepsilon(n)=\frac{1}{2} \sum_{k} e_{k}^{2}(n)
$$

Use the steepest gradient descent method to get:

$$
\Delta w_{k j}(n)=\eta(n) e_{k}(n) x_{j}(n)
$$

The NN model that this kind of learning rule is successfully applied is very extensive, such as the simplest perceptron learning algorithm, which is also the most primitive application of NN. In the competitive learning of the network, the output units compete with each other, and finally, only one of the strongest can be activated. The rule is expressed by the following formula:

$\Delta w_{k j}(n)=\left\{\begin{array}{l}\eta\left(x_{j}-w_{j i}\right), \quad \text { If the neuron competition wins } \\ 0, \quad \text { If the neuron competition fails. }\end{array}\right.$

In practical application, we all adopt the fuzzy comprehensive evaluation model to judge many problems; among which, if there are many factors, we adopt the multilevel fuzzy comprehensive evaluation method of layering the factor set. For example, in the comprehensive evaluation of an enterprise, the evaluation factors are firstly divided into several categories, such as the hardware equipment of the school, the strength of teachers, and the development of the school, and then, the hardware equipment is evaluated. The multilevel fuzzy comprehensive evaluation method is the process of judging each category of factors separately and then making overall evaluation. After the hierarchical division, the relationship between various factors is clearer, and the situation that the weight of factors is too small due to the normalization of weight is greatly avoided [30]. In the evaluation of employees' psychological health, we propose a multifactor fuzzy comprehensive decision-making model, that is, a multilevel comprehensive fuzzy evaluation model. If the learning system is in a stable working state, the statistical characteristics of the environment can be learned through supervised learning, which can be remembered by the NN as experience. If the environment is unstable, the usual supervised learning cannot track this change. To solve this problem, the network needs some adaptive ability. The algorithm of deep BP network in this paper includes two propagation directions: forward propagation of input signal and backward propagation of output error, that is to say, the actual network output is calculated from input to output. However, the weights and thresholds of each layer of the network are modified in the reverse direction from output to input (as shown in Figure 3).

The weight of the connection between each node represents the constraint between each hypothesis. For each node, there can be input from the outside, which reflects external evidence.

\section{Result Analysis and Discussion}

The degree of network convergence in the training of $\mathrm{NN}$ is determined by the quality of data. In the shortest amount of time, good data can help the network achieve a better convergence balance. On the other hand, regardless of how network's parameters are changed, it is difficult to achieve the desired results if the data is incorrect. The input and output data of the NN should be preprocessed first in order to make NN's training more effective and improve the training speed of the established NN [31]. The sample data is divided into two parts at random: one part is used as training samples to train the network, and the other part is used as test samples to determine whether the trained network meets the requirements. Finally, the model is simulated and tested to see if it can achieve the desired prediction goal. The error of the network is constantly decreasing in the process of self-learning, as shown by the curve description in Figure 4. The network can basically achieve the minimum error prediction through continuous training of the NN, and it can be seen that the prediction model has a good prediction effect. The network can basically achieve the minimum error prediction through continuous NN training.

After "learning" the sample set, the weights and thresholds used by the $\mathrm{NN}$ are the internal representations obtained by the NN through adaptive learning that best 


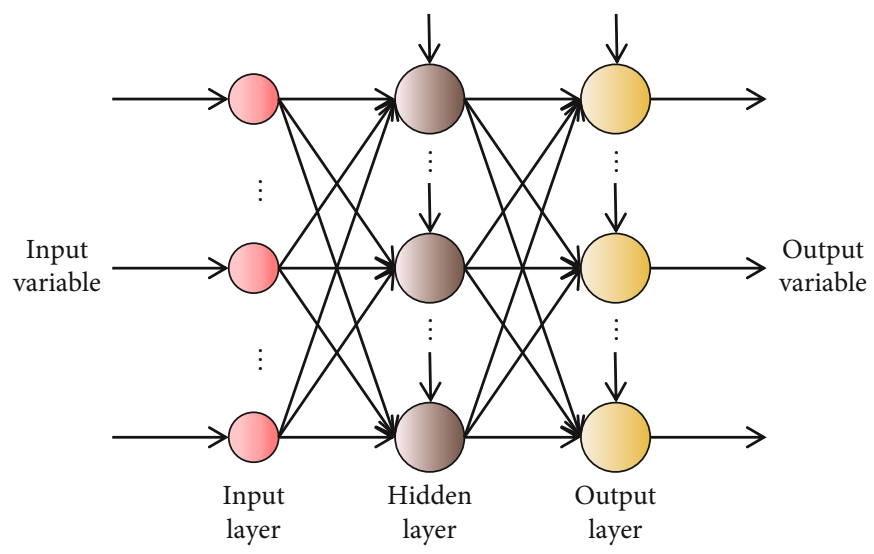

FIGURE 3: NN structure.

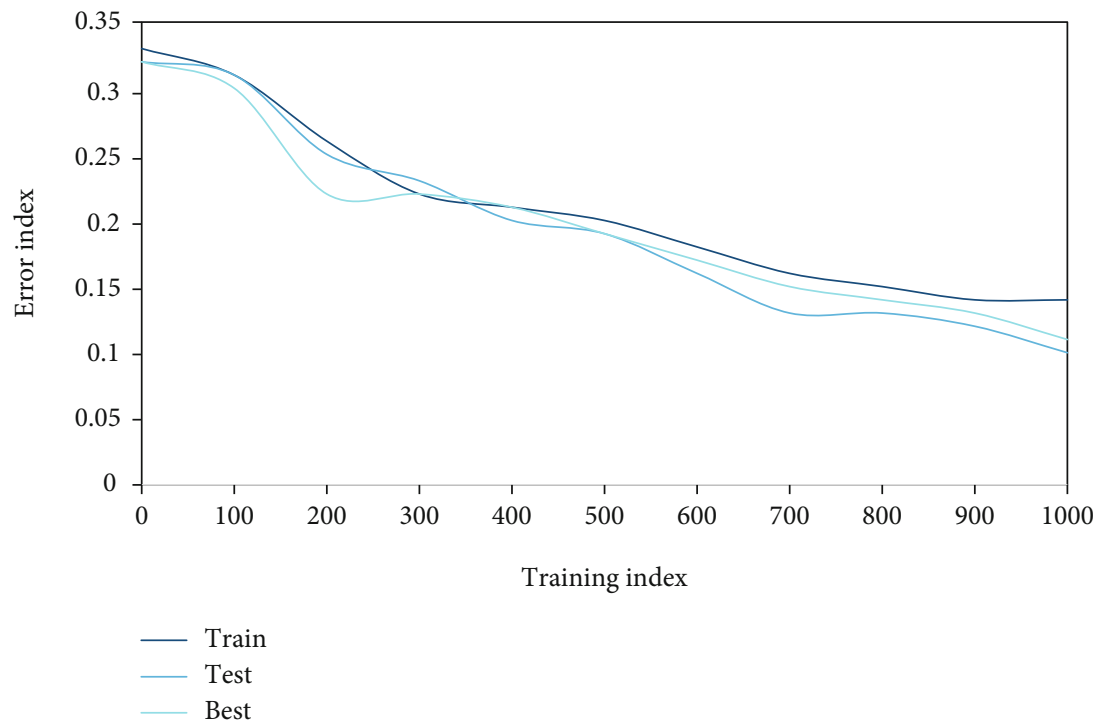

FIGURE 4: NN training process.

meet the data requirements. Then, the feature data of the sample set to be identified is input into the trained network, and the network can automatically reason and identify the output results of the samples. Figure 5 shows the comparison of errors before and after optimization. It can be found that the optimized NN prediction model is better than the NN before optimization.

The data fitting effect can be achieved faster with the optimised NN. The ability of the optimised NN to fit data is clearly improved without changing the original network structure and network parameters. The reason for this is that the genetic algorithm uses the fitness function to determine the optimal weights and thresholds and then gives those weights and thresholds to the $\mathrm{NN}$ for training, rather than using random weights and thresholds generated by Matlab for each training, which greatly reduces the weights and thresholds' ability to self-adjust. The number of iterative steps in the NN is significantly reduced after optimisation, and the preset goal can be reached much faster. Figure 6 depicts the difference between the BP network's expected output and model's actual output.

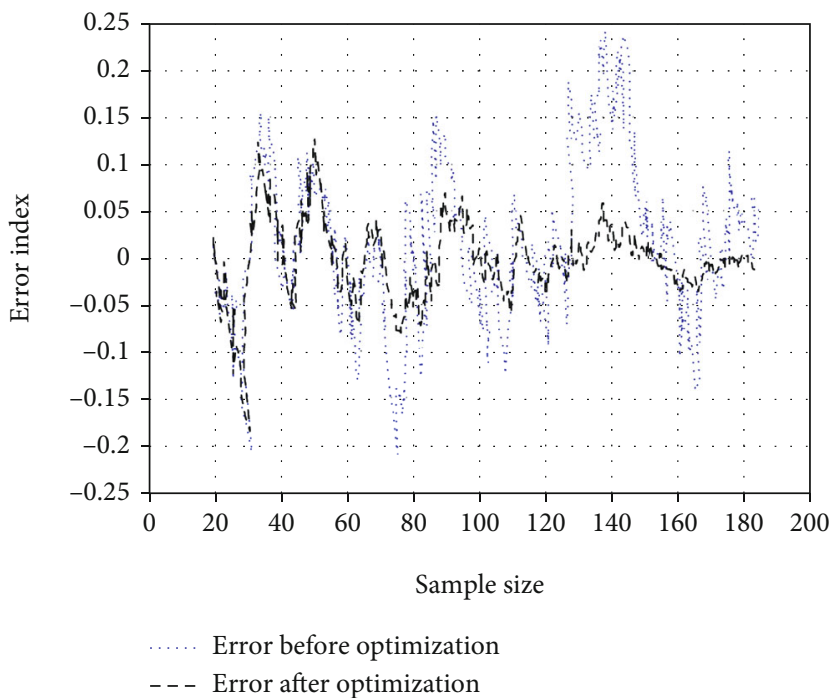

FIGURE 5: Error comparison before and after optimization. 


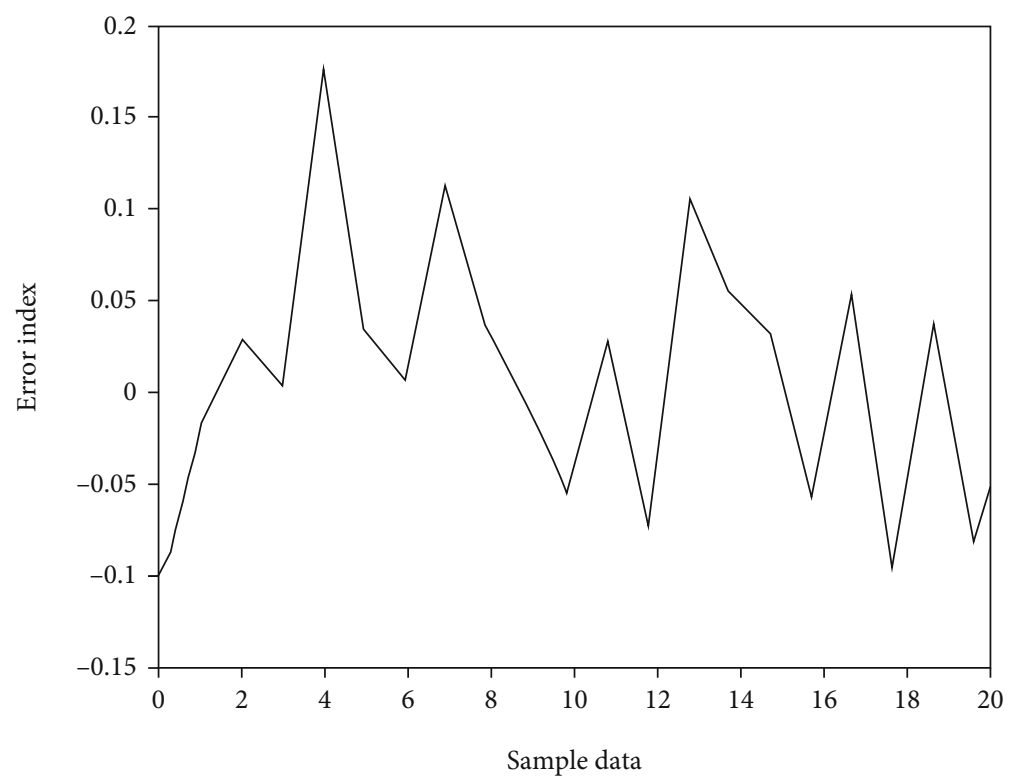

FIgURE 6: The error between the expected output of the BP network and the actual output value of the model.

Figure 7 shows the dynamic evaluation effect of employees' psychological health. Generally speaking, DNN can effectively evaluate the dynamic trend of employees' psychological health in a period of time, regardless of the training set and verification set in the sample or the test set outside the sample. It proves the applicability and effectiveness of the DNN in evaluating the marketing situation.

In the $\mathrm{NN}$ analysis model, the concept of state transition is introduced, so that the probability of transition from one state to another can be obtained, and the future can be judged, so as to evaluate and guide the future work. Describing psychological phenomena with mathematical models has the advantages that it is not only more general, accurate, deductive, and predictive than the description with natural language, but more importantly, it is convenient for computer simulation and creates conditions for the development of artificial intelligence. However, computer's ability to identify fuzzy phenomena is poor. In order to improve computer's ability to identify fuzzy phenomena, it is necessary to design the fuzzy language commonly used by people into instructions and programs that can be accepted by the machine, so that the machine can make corresponding judgments as succinctly and flexibly as the human brain, thus improving the efficiency of automatically identifying and controlling fuzzy phenomena.

The trained network is simulated with sim () function, and then, the simulated results are denormalized and compared with the original data. The result of comparing the predicted data with the real data is shown in Figure 8.

It can be seen from Figure 8 that the obtained simulation data is very close to the real data. This shows that after training, BP network has a good approximation effect on the prediction of employees' psychological status. In this paper, the quantitative data of the factors affecting the psychological health of enterprise employees are mapped to the corresponding psychological health status, and the prediction model of enterprise employees' psychological health is established. The results show that the prediction model can make a good prediction for students with psychological problems through the quantification of various influencing factors by employees and basically achieve the expected goal.

The complexity of psychological phenomena is selfevident. At present, the evaluation and evaluation methods of psychological health are still in the stage of discussion. Factors affecting psychological health are all fuzzy, and it is difficult to have a strict standard when determining the degree of influence of some information collected by various scales on psychological health. There is also a certain degree of relative ambiguity when defining the three states of psychological health, mental unhealthy, and mental illness. The psychological state of each individual is a multidimensional information system, which is characterized by multivariable and strong coupling, and complex nonlinear interactions among various factors in the system. Estimation, due to the nonlinear relationship of various factors, is difficult to describe by traditional mathematical methods, but it is more suitable to use systematic analysis to evaluate psychological health, considering the relationship between input and output of the evaluation system. At the social level, the first step of psychological health education is to do a good job of publicity, create an atmosphere that attaches importance to psychological health in the whole society, and do a good job of publicity and popularization of psychological health and psychological health knowledge through the Internet, radio, television, newspapers, magazines, and various publicity tools. Secondly, it is necessary to establish a network system of psychological health education covering the whole society, so that employees of psychological health education can exchange information and cooperate with each other to realize resource sharing. 


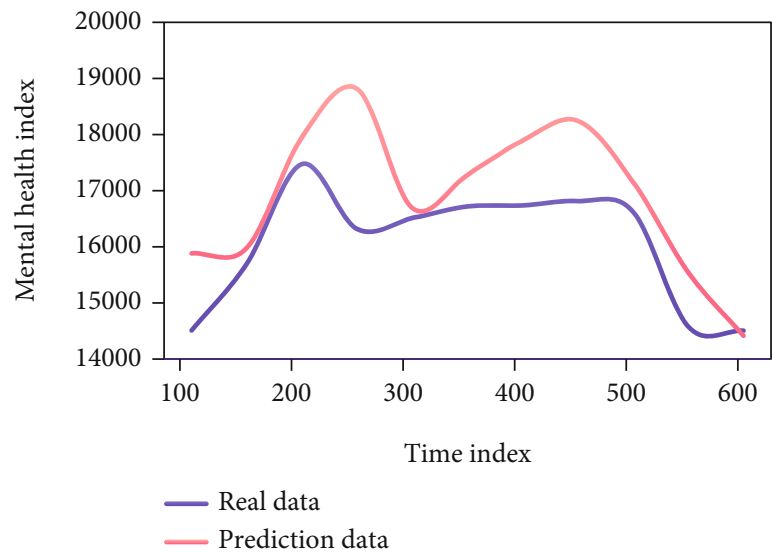

(a)

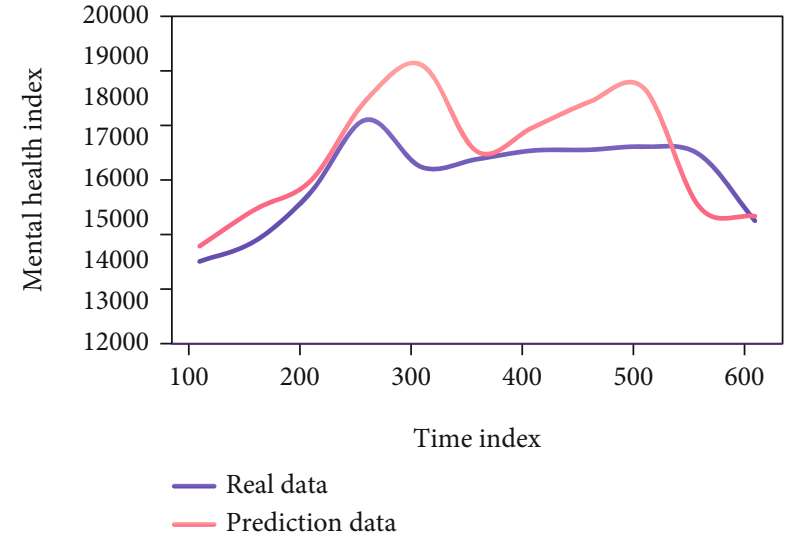

(b)

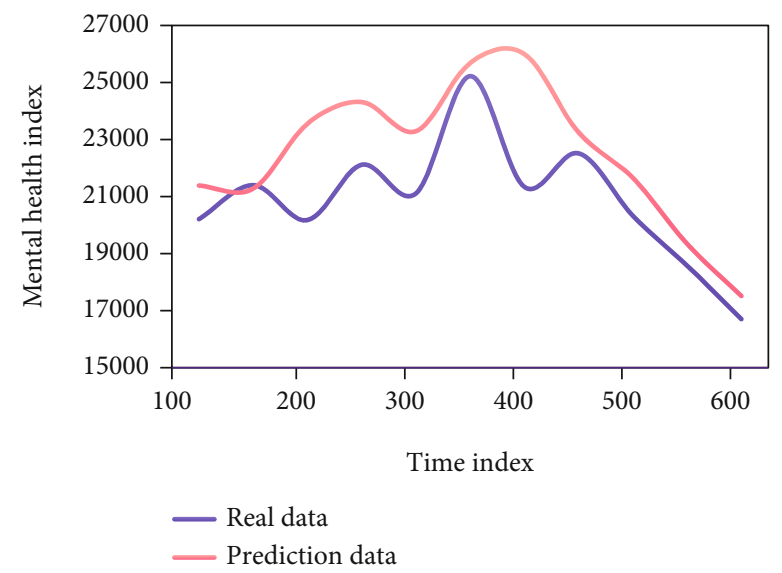

(c)

Figure 7: (a) Dynamic evaluation effect (training set). (b) Dynamic evaluation effect (validation set). (c) Dynamic evaluation effect (test set).

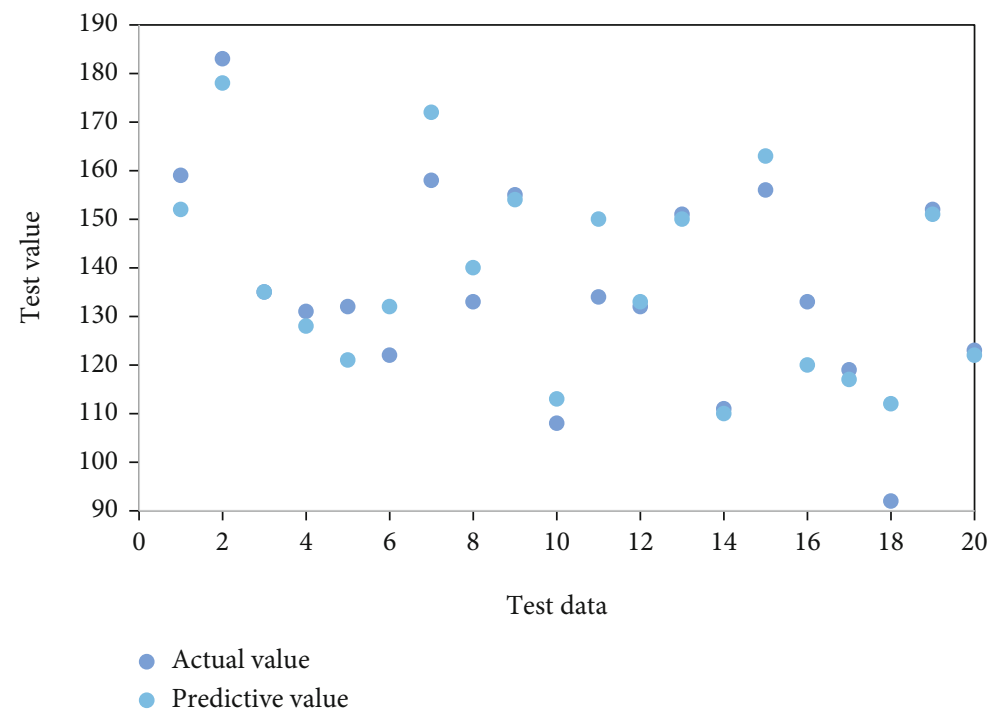

Figure 8: Graphical output of the network after training.

\section{Conclusions}

The entire society must pay attention to social individuals' psychological health problems, place a high value on, strengthen, and improve existing psychological health education methods and methods, and conduct psychological health education for people at all levels, including psychological concept, family life, work environment, 
and social culture, in order to promote the improvement of social individual psychological quality and enhance the harmony. In comparison to traditional employee psychological health prediction methods, the DNN prediction method avoids the time-consuming and uncertain traditional modelling process. This paper develops a mathematical prediction network model based on the DNN principle and an analysis of the psychological factors affecting employees. The calculation of the final result of the prediction system is simple and flexible when the parameters of the $\mathrm{NN}$ are changed, and the network model's prediction efficiency and accuracy are greatly improved. This, to a large extent, provides a good reference method for the study of enterprise employees' psychological health.

The multilayer feedforward DNN used in this prediction model still has some flaws, such as a slow learning rate, a tendency to fall into a local minimum during training, and a contradiction between the depth network's training and prediction abilities. Other resilience qualities, such as emotional intelligence for service employees and cultural intelligence for foreign-funded enterprises, may be required to deal with occupational adversity. As a result, in future research, the research field must be continuously expanded and explored in order to improve the research in this field. The whole society must pay attention to the mental health problems of social individuals, attach great importance to, strengthen and improve the existing methods of mental health education, and educate people on mental health from various aspects such as psychological concept, family life, working environment, and social culture, so as to promote the improvement of social individuals' psychological quality and the harmony of interpersonal relationships, thus enhancing social trust and harmony. The factors that influence the mental health of enterprise employees change with time, and the main factors that influence the mental health of enterprise employees will also change accordingly. This paper gives a detailed description of how to use the theory and method of deep neural network and fuzzy mathematics to establish a mathematical model to evaluate the mental health status, and how to improve the accuracy of the model evaluation by combining the scale measurement tools applied to psychological evaluation with reality. In the process of safe production in enterprises, employees' mental health problems are deeply influenced by the enterprises themselves, society and employees. It is necessary to fully guarantee all aspects of enterprises to work together, establish people-oriented management ideas, and create a good production environment. I believe that in the near future, neural networks will be applied to more and more aspects, and people's lives will be more convenient through deep neural networks.

\section{Data Availability}

The data used to support the findings of this study are included within the article.

\section{Conflicts of Interest}

The author declares that there are no conflicts of interest.

\section{References}

[1] W. Yuxing, H. Jun, and P. Yingjie, "Application of GA-BP NN in the prediction of elderly negative emotions," Small Microcomputer System, vol. 41, no. 8, pp. 136-140, 2020.

[2] S. Songhe, F. Shaozhen, Z. Zhimin, and G. Zhong, "Effect analysis of psychological intervention for middle-aged and elderly immigrants in the south-to-north water transfer project," Modern Preventive Medicine, vol. 43, no. 6, pp. 1051-1056, 2016.

[3] Y. Jing, W. Yong, and S. Lili, "Research progress on the neural mechanism of mental resilience," Journal of Neuroanatomy, vol. 35, no. 1, pp. 94-98, 2019.

[4] D. Arifoglu and A. Bouchachia, "Detection of abnormal behaviour for dementia sufferers using convolutional neural networks," Artificial Intelligence in Medicine, vol. 94, no. 3, pp. 88-95, 2019.

[5] A. Shatte, D. M. Hutchinson, and S. J. Teague, "Machine learning in mental health: a scoping review of methods and applications," Psychological Medicine, vol. 49, no. 9, pp. 1426-1448, 2019.

[6] R. Ramírez-Barrantes, I. Marchant, and P. Olivero, "TRPV1 may increase the effectiveness of estrogen therapy on neuroprotection and neuroregeneration," Neural Regeneration Research, vol. 11, no. 8, pp. 1204-1207, 2016.

[7] F. Akram and J. Giordano, "Research domain criteria as psychiatric nosology," Cambridge Quarterly of Healthcare Ethics, vol. 26, no. 4, pp. 592-601, 2017.

[8] M. Zin, S. Ismail, and K. Karuppiah, "Smart Co2 detector prototype development to enhance the efficiency of ventilation system in a building," Iranian Journal of Public Health, vol. 45, no. 1, pp. 93-99, 2016.

[9] S. Rohajawati, D. I. Sensuse, Y. G. Sucahyo, and A. M. Arymurthy, "Mental health knowledge management: critical success factors and strategy of implementation," Journal of Knowledge Management, vol. 20, no. 5, pp. 980-1003, 2016.

[10] D. Xiaona, "Application of "focus on short-term psychological consultation" in mental health education of enterprise employees," Early Education and Development, vol. 5, no. 2015-4, pp. 49-50, 2021.

[11] Z. Yong, Y. Ding, and L. Jia, "The mental health status of employees in small and medium-sized science and technology enterprises in Hubei Province and its promotion countermeasures," Industrial Safety and Environmental Protection, vol. 45, no. 3, pp. 45-48, 2019.

[12] L. Jie, "Analysis on how to strengthen the mental health management of employees in state-owned enterprises," Journal of the Party School of the Taiyuan Municipal Committee of the Communist Party of China, vol. 198, no. 1, pp. 72-73, 2020.

[13] A. Jordan, M. L. Mathis, and J. Isom, "Achieving mental health equity: addictions," Psychiatric Clinics of North America, vol. 43, no. 3, pp. 487-500, 2020.

[14] M. Rocha, M. Marin, and J. Macias-Seda, "Condições de vida, trabalho e saúde mental: um estudo com trabalhadores brasileiros e espanhóis que atuam em serviço de limpeza hospitalar," Ciência \& Saúde Coletiva, vol. 25, no. 10, pp. 38213832, 2020. 
[15] R. Clarke, J. Jeffrey, M. Grossman, T. Strouse, M. Gitlin, and S. A. Skootsky, "Delivering on accountable care: lessons from a behavioral health program to improve access and outcomes," Health Affairs, vol. 35, no. 8, pp. 1487-1493, 2016.

[16] W. Ning, W. Jinyan, and L. Fei, "The influence of emotion on time perception and its neurophysiological mechanism," Acta Physiologica Sinica, vol. 68, no. 4, pp. 464-474, 2016.

[17] S. Kalfa, L. Branicki, and S. Brammer, "Organizational accommodation of employee mental health conditions and unintended stigma," The International Journal of Human Resource Management, vol. 32, no. 15, pp. 3190-3217, 2021.

[18] K. Snyder, M. Hill, M. Lee, T. N. Crawford, and M. Orlowski, "The relationships between physical health and chronic disease, stress, and resource strain in head start employees," Workplace Health \& Safety, vol. 68, no. 4, pp. 190-201, 2020.

[19] A. Luqman, S. Talwar, A. Masood, and A. Dhir, "Does enterprise social media use promote employee creativity and wellbeing?," Journal of Business Research, vol. 131, no. 2, pp. 40$54,2021$.

[20] M. Sarrias and B. Jara, "How much should we pay for mental health deterioration? The subjective monetary value of mental health after the 27F Chilean earthquake," Journal of Happiness Studies, vol. 21, no. 3, pp. 843-875, 2020.

[21] A. Dovran, D. Winje, S. Øverland, K. Arefjord, A. Hansen, and L. Waage, "Childhood maltreatment and adult mental health," Nordic Journal of Psychiatry, vol. 70, no. 2, pp. 140-145, 2016.

[22] Y. Bin, "An empirical study on the relationship between work pressure and the psychological health of tea enterprise employees," Fujian Tea, vol. 39, no. 8, pp. 306-307, 2017.

[23] X. Mingjin, H. Xianni, F. Zhiyuan, and Y. Yang, "The mediating effect of coping styles in the relationship between work stress and mental health of nuclear enterprise employees," Environmental and Occupational Medicine, vol. 33, no. 2, pp. 134-138, 2016.

[24] J. Kong, H. Wang, X. Wang, X. Jin, X. Fang, and S. Lin, "Multistream hybrid architecture based on cross-level fusion strategy for fine-grained crop species recognition in precision agriculture," Computers and Electronics in Agriculture, vol. 185, article 106134, 2021.

[25] J. Xue, X. Gu, and T. Ni, "Auto-weighted multi-view discriminative metric learning method with fisher discriminative and global structure constraints for epilepsy EEG signal classification," Frontiers in Neuroscience, vol. 14, 2020.

[26] Y. Y. Zheng, J. L. Kong, X. B. Jin, X. Y. Wang, and M. Zuo, "CropDeep: the crop vision dataset for deep-learning-based classification and detection in precision agriculture," Sensors, vol. 19, no. 5, p. 1058, 2019.

[27] M. J. Kaas, "Will we be ready? Preparing psychiatric-mental health nurses for future practice," Journal of the American Psychiatric Nurses Association, vol. 26, no. 1, pp. 112-119, 2019.

[28] G. Fairchild, "Mind the gap: evidence that child mental health inequalities are increasing in the UK," European Child \& Adolescent Psychiatry, vol. 28, no. 11, pp. 1415-1416, 2019.
[29] C. M. Tucker, J. Roncoroni, and L. P. Buki, "Counseling psychologists and behavioral health: promoting mental and physical health outcomes," The Counseling Psychologist, vol. 47, no. 7, pp. 970-998, 2019.

[30] X. Tang, L. Yang, and Z. Miao, "Research on the relationship between coping style and mental health of employees in a pharmaceutical company's assembly line in Chengdu," China Medical Herald, vol. 542, no. 12, pp. 78-81, 2020.

[31] L. Pengfei, L. Lei, C. Tingting, and M. Zhang, "Investigation on occupational stress and mental health of employees in a glove manufacturer," Industrial Hygiene and Occupational Diseases, vol. 45 , no. 6 , pp. 55-59, 2019. 